\title{
Chemical Sensing Sensitivity of Long-Period Grating Sensor Enhanced by Colloidal Gold Nanoparticles
}

\author{
Jaw-Luen Tang ${ }^{1, *}$ and Jien-Neng Wang ${ }^{2}$
}

1 Department of Physics, National Chung Cheng University, 168 University Road, Chia-Yi 621, Taiwan; Tel.: +886-5-272-0586; Fax +886-5-272-0587; E-mail: phyjlt@ccu.edu.tw.

2 Department of Construction Engineering, National Yunlin University of Science and Technology, Yun-Lin 640, Taiwan; Tel.: +886-5-534-2601-4723, Fax +886-5-531-2049; E-mail: wangjn@yuntech.edu.tw

* Author to whom correspondence should be addressed.

Received: 13 November 2007 / Accepted: 4 January 2008 / Published: 21 January 2008

\begin{abstract}
A simple and effective method is proposed to improve spectral sensitivity and detection limit of long period gratings for refractive index or chemical sensing, where the grating surface is modified by a monolayer of colloidal gold nanoparticles. The transmission spectra and optical properties of gold nanospheres vary with the different refractive index of the environment near the surface of gold nanospheres. The sensor response of gold colloids increases linearly with solvents of increasing refractive index. The results for the measurement of sucrose and sodium chloride solutions are reported, which show that this type of sensor can provide a limiting resolution of $\sim 10^{-3}$ to $\sim 10^{-4}$ for refractive indices in the range of 1.34 to 1.39 and a noticeable increase in detection limit of refractive index to external medium.
\end{abstract}

Keywords: Spectral sensitivity; refractive index; long-period grating; colloidal gold nanoparticles; localized surface plasmon resonance.

\section{Introduction}

Recently, long period fiber grating (LPFG), which is produced by introducing a periodic modulation of the refractive index (RI) in the core of a single-mode fiber, has rapidly emerged as an 
excellent RI sensor for chemical and biochemical sensing. An LPFG allows light coupling between the fundamental mode and the cladding modes in the same direction, which results in several attenuation bands centered at discrete wavelengths in the transmission spectrum [1]. LPFGs have several favorable features including low insertion losses, low background back-reflection, polarization independence, high temperature sensitivity, and relatively simple fabrication. These gratings have been offered many useful applications in the fields of optical fiber communication and fiber sensing such as wavelength filters [1], gain flattening components [2], dispersion compensators [3], widely tunable filters [4], broadband add/drop multiplexers [5], and various types of optical sensors [6-12]. The LPFG is extremely sensitive to the RI of the medium surrounding the cladding region, thus allowing it to be employed as an ambient RI sensor. At the same time, there has been an increasing interest in applying the extraordinally optical properties (e.g., absorbance and resonance wavelength) of noble metal colloidal nanoparticles such as $\mathrm{Au}$ or $\mathrm{Ag}$, due to their potential applications for chemical and biological sensing. A number of nanoscale biosensors and chemosensors have been realized through shifts in the localized surface plasmon resonance (LSPR) extinction maximum of gold or silver nanoparticles [13-16]. The wavelength shifts are mainly caused by adsorbate-induced local RI changes at the surfaces of nanoparticles. Among the LSPR sensors, we have recently demonstrated an optical fiber biosensor that exploits the LSPR of self-assembled Au colloids (SAGC) on the grating portion of a long period fiber grating [17]. The attenuated total reflection spectrum of SAGC is sensitive to the RI of its surrounding medium, which can be used for monitoring the solution bulk and for label-free detection of antigen/antibody binding at the surface of the Au colloids. The advantages of this type of sensor are their simple fabrication and ease of use. Moreover, the sensor has the potential capability for on-site and remote sensing, can be easily multiplexed to enable high-throughout screening of bimolecular interactions, and has the potential use for disposable sensors.

This paper presents a simple and low-cost chemosensor utilizing the modification of the grating portion of an LPFG with aqueous solution of chemicals under test, which is sensitive to the RI of the cladding surface and, hence, is suitable for detecting chemical solutions. The study of LPFG-based sensors and their possible applications in chemical sensing is presented. Using sucrose solution and sodium chloride solution as examples, we demonstrate that substantial enhancement of RI sensitivity and limit of detection for chemical solution concentration sensing can be achieved by using an LPFG sensor with its cladding surface modified by gold nanoparticles.

\section{Principle of refractive index sensing}

The LPFG with periods ranging from several hundred microns to several millimeters couples incident light guided by a fundamental mode in the core to different forward-propagating cladding modes of high diffraction order $m$ in an optical fiber, which decay rapidly due to the radiation from scattering losses. The coupling of the light into the cladding region generates a set of resonant bands centered at wavelength $\lambda_{m}$ in the transmission spectrum of the fiber. The resonance wavelengths $\lambda_{m}$ of an attenuation band are solutions of the following phase matched conditions [12]:

$$
\lambda_{m}=\left[n_{c o}^{e f f}-n_{c l, m}^{e f f}\right] \Lambda=\delta n_{e f f} \Lambda
$$


where $\Lambda$ is the period of grating, and $n_{c o}^{e f f}$ is the effective RI of the fundamental core mode at the wavelength of $\lambda_{m}$, which is also dependent on the core RI and cladding RI. Also $n_{c l, m}^{\text {eff }}$ is the effective RI of the $m$ th radial cladding mode $(m=2,3,4, \ldots)$ at the wavelength $\lambda_{m}$, which also is a function of cladding RI and in particular the RI of the surrounding medium $n_{s}$. Note that both indices are dependent on the temperature and the strain experienced by the fiber. The spectral properties of individual cladding modes are determined by the fiber structure and may be observed through their associated attenuation bands. When the concentration or the RI of the surrounding medium changes also $n_{c l, m}^{e f f}$ changes and a wavelength shift can be obtained in the transmission spectrum. The wavelength shift can be linearly related to the concentrations of the solution under test. An LPFG can be very sensitive to the changes in temperature and deformations by fiber imperfections, loading, and bending, which also produce a noticeable wavelength shift in loss peaks. Therefore, to precisely measure variations of concentrations or RI changes, temperature changes and deformations must be compensated or avoided.

Since the grating period is unaltered under the effect of a change in ambient RI and assuming that the RI of the core mode remains unchanged by $n_{s}$, the influence of variation in the RI around the cladding of an LPFG is expressed by:

$$
\left(\frac{d \lambda}{d n_{s}}\right)_{m}=\left(\frac{d \lambda}{d n_{c l, m}^{e f f}}\right)\left(\frac{d n_{c l, m}^{e f f}}{d n_{s}}\right)
$$

The spectral sensitivity, defined as $\frac{d \lambda}{d n_{s}}$, is relevant to each of the measurands and contributes to effective wavelength change of the $m$ th cladding mode. The RI sensitivity of an LPFG arises from the dependence of the resonance wavelength on the effective RI of cladding mode, which also is dependent on the RI of the surrounding materials. The LPFG is expected to have a strong dependence on the order of the coupled cladding mode since for each cladding mode $\frac{d n_{c l, m}^{e f f}}{d n_{s}}$ is distinct. In general, as $n_{s}$ increases, the spectral sensitivity increases monotonically to a maximum values, which occurs at the value of $n_{s}$ at which the cladding mode becomes unguided. The wavelength shift arising from the RI changes, for a given fiber and cladding mode, may be positive or negative, depending on the local slope of characteristic phase-matching curve $d \lambda / d \Lambda$. Fig. 1 illustrates a typical measured transmission spectrum of an LPFG in air and in de-ionized water. Fig. 2 shows the wavelength shift in one of the resonance bands with respective to $n_{s}$ for an LPFG written in a Furukawa SM-332 fiber. The shift is negative for all band and increases with the order of the cladding mode for this grating. It is shown that the LPFG's RI sensitivity is predominantly within the range of 1.33 to 1.46 . The distinct resonance bands disappear at approximately $n_{s}=1.46$ when the cladding mode converted to radiation mode losses. The maximum RI sensitivity is imposed by the RI of the cladding material. No measurable wavelength shift is observed for RI above this limit. The highly sensitivity of the LPFG to the surrounding medium has offered the potential to monitor chemical changes and corrosion condition for various embedded material structures. The sensitivity of an LPFG to RI changes can be enhanced of reduced by 
controlling the fiber parameters and the grating period for coupling to specific cladding modes. Previously we have illustrated the feasibility of making this colloidal gold modified LPFG for chemical and biochemical sensing. In this paper, we extend the study and aim to demonstrate that the spectral sensitivity can be enhanced when the grating surface of an LPFG is modified by colloidal gold nanoparticles.

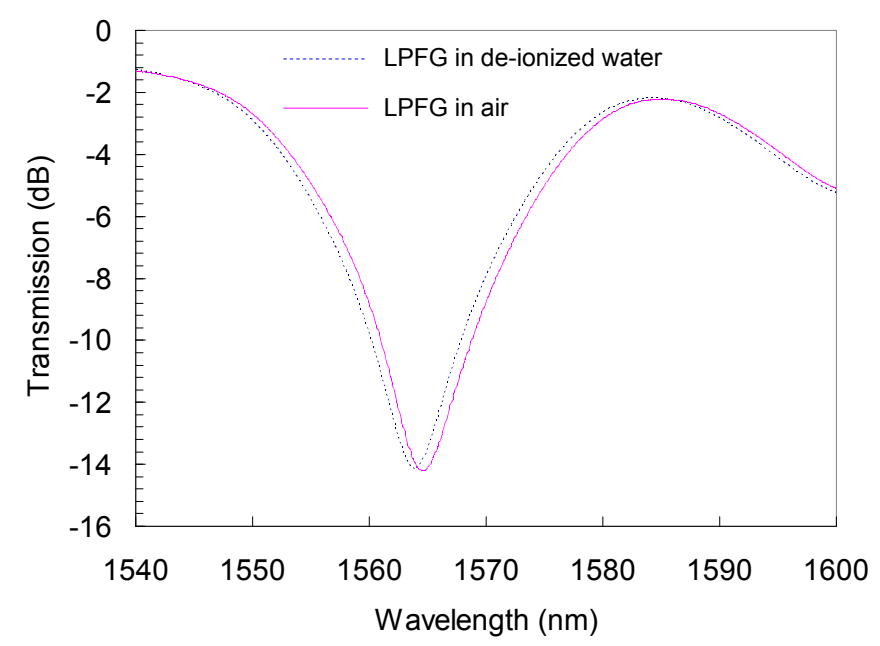

Figure 1. Transmission spectrum of an LPFG in air (solid line) and in de-ionized water (dash line).

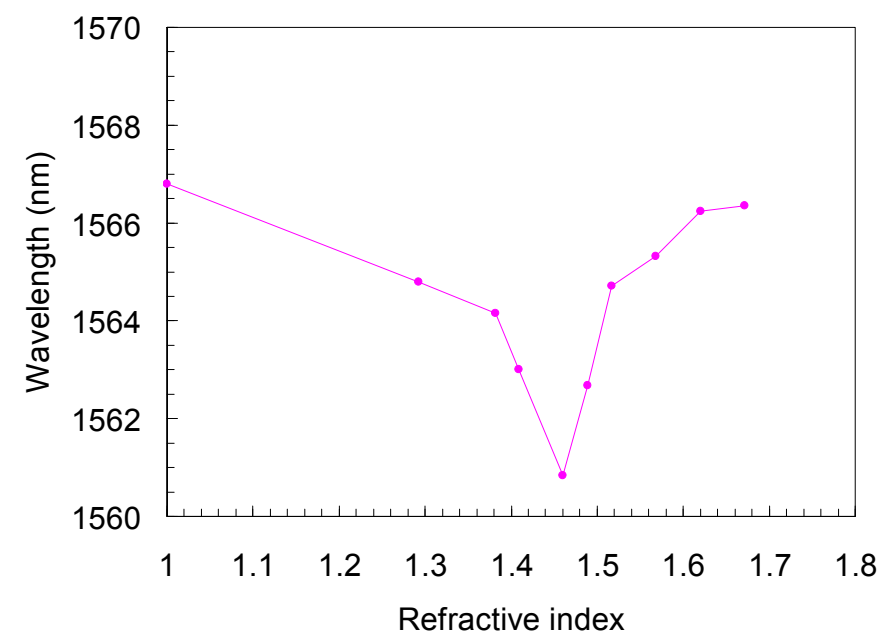

Figure 2. Wavelength shifts in one of the resonance bands of an LPFG with refractive index changes.

\section{Experimental Section}

The LPFGs used in this experiment were fabricated in a single-mode fiber using a high-frequency $\mathrm{CO}_{2}$ laser system through the point-to-point technique, a simple and highly controllable method for inducing periodic index changes on a bare fiber $[17,18]$. The experimental setup is shown in Fig. 3, which is consisted of a computer-controlled $\mathrm{CO}_{2}$ laser (SYNRAD 48-2, $25 \mathrm{~W}$ full power) associated with a high-speed laser beam scanner, a fiber holder, a broadband light source, and an optical spectrum 
analyzer. A translation stage is used to position the fiber in the alignment fixture. The beam scanner is constructed by a galvanometer mirror and F-theta lenses, which moves the focused laser bean with a $290 \mu \mathrm{m}$ beam spot across a field size of $110 \mathrm{~mm}$. The two-beam position resolution of the system is about $30 \mu \mathrm{m}$ and the maximum speed of moving successive beam pairs could be up to $40 \mathrm{kHz}(25 \mu \mathrm{s})$. A broadband light source $(600-1700 \mathrm{~nm})$ and a high-resolution spectrum analyzer are employed to insitu analyze the transmission loss as the grating was written.

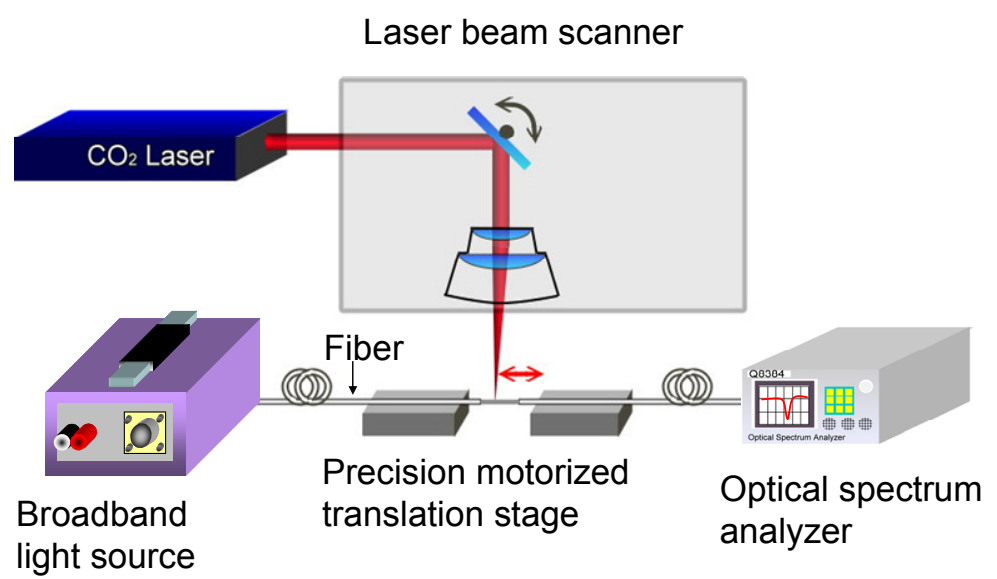

Figure 3. Schematic of experimental set-up for LPFG fabrication using $\mathrm{CO}_{2}$ laser pulses.

The LPFGs (period $550 \mu \mathrm{m}$, length $2.5 \mathrm{~cm}$ ) studied in this work were fabricated in Furukawa SM332 or Corning SMF-28 fibers with a laser power of $0.8 \mathrm{~W}$ and a total exposure time of about $2 \mathrm{~min}$. The transmission spectrum was interrogated during the writing and its characteristics such as insertion loss, resonance peak wavelength, and peak depth were analyzed after the grating was written. With suitable fabrication parameters such as laser power, exposure time, grating period, and laser scan speed, the resulting resonance wavelengths ranging from $1200 \mathrm{~nm}$ to $1600 \mathrm{~nm}$ with a greater than 20 $\mathrm{dB}$ peak depth were obtained. The fabrication time was less than $2 \mathrm{~min}$ for a typical $2.5 \mathrm{~cm}$ long LPFG. After the laser exposure, all the LPFGs were annealed at $150{ }^{\circ} \mathrm{C}$ for $24 \mathrm{~h}$ to stabilize their transmission spectrum.

Here, the procedure for preparation of gold colloids has been reported elsewhere [13-15]. Transmission electron microscopic (TEM) image analysis (Fig. 4) shows that the mean diameter of the gold colloids was $8.4 \pm 2.8 \mathrm{~nm}$. The average surface coverage (relative to a close-packed monolayer) of a self-assembled colloid monolayer of gold $\left(\mathrm{CM}_{\mathrm{Au}}\right)$ was estimated to be $30 \%$ by atomic absorption spectroscopy. The self-assembled $\mathrm{CM}_{\mathrm{Au}}$ 's on glass substrate were characterized by UV-vis spectrophotometry. We have optimized the process time of self-assembled for gold colloids on glass substrate to obtain a reasonable absorbance for further applications. Fig. 5 is the absorption spectrum of $\mathrm{CM}_{\mathrm{Au}}$ 's on glass prepared by three different self-assembled time, which are $20 \mathrm{~min}, 23 \mathrm{~min}$ and 25 min, respectively. The self-assembled time of $23 \mathrm{~min}$ was chosen for it achieved the maximum absorbance. The preparation of colloidal Au-modified long-period fiber gratings (or $\mathrm{CM}_{\mathrm{Au}} \mathrm{LFPG}$ ) [17] 
is adopted from the same procedure as that of the colloidal Au-modified optical fibers $\left(\mathrm{CM}_{\mathrm{Au}} \mathrm{OF}\right)$ in Ref. 16. Since the fiber used in this study is an optical fiber whose core and cladding are made of silica glass, one would expect that, when the concentration of surrounding medium changed, self-assembled $\mathrm{CM}_{\mathrm{Au}}$ 's on glass substrate will exhibit similar behavior such as LSPR maximum and absorbance changes as compared to those self-assembled $\mathrm{CM}_{\mathrm{Au}}$ 's on the cladding portion of a fiber. Furthermore, both LSPR maximum and absorbance changes depend on the mean size and density of our gold colloids monolayer, which can be controlled by controlling formation conditions of gold colloids such as temperature, time and the concentration of the solution. Previous publication as in Ref. 13 has shown that the particle density of gold colloids monolayer and, hence, the mean diameter and absorption of the gold colloids, can be controlled by varying the immersion time (or self-assembled time) in the colloidal gold suspension. Hence, here one can adopt the analogous reasoning to our LPFG test samples in determining the optimized mean diameter and absorption of the gold colloids through controlling the self-assembled time. A self-assembled colloid monolayer of gold on the grating surfaces of LPFG was used for further study.

The experimental set-up to measure spectral sensitivity of this sensor for chemical sensing is adopted from Ref. 17, which includes a broadband light source, a sensing LPFG fiber, a cell, and an optical spectrum analyzer. The LPFG sensor was immersed in different media such as air, water and sucrose solutions and its transmission spectrum was measured with the optical spectrum analyzer. Each transmission spectrum was referenced to the background spectrum of a bare fiber in air. In this work, a number of LPFGs and $\mathrm{CM}_{\mathrm{Au}}$ LPFGs grating sensors associated with various attenuation bands were measured and investigated.

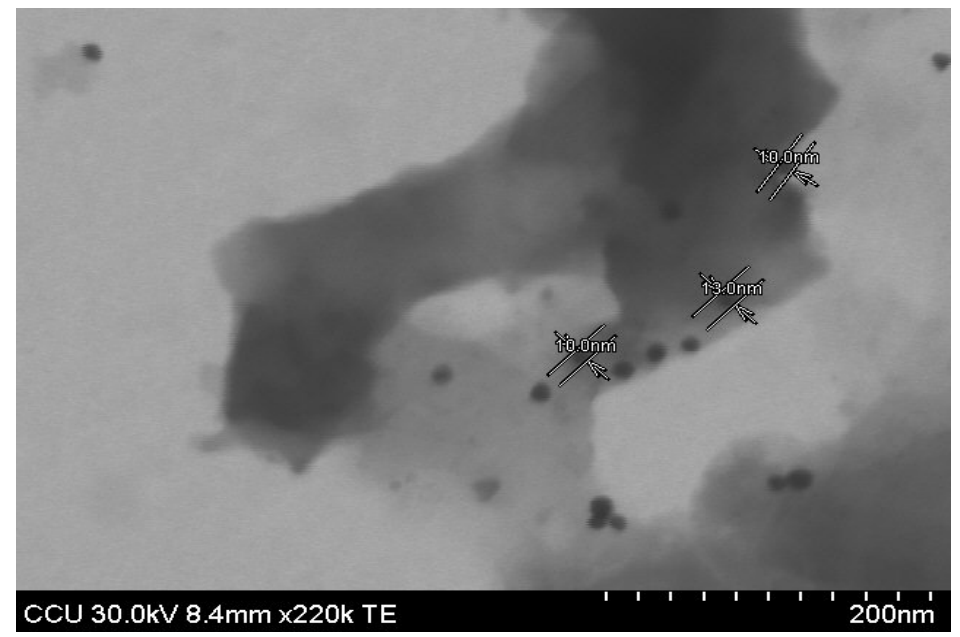

Figure 4. TEM image of colloid gold nanospheres. 


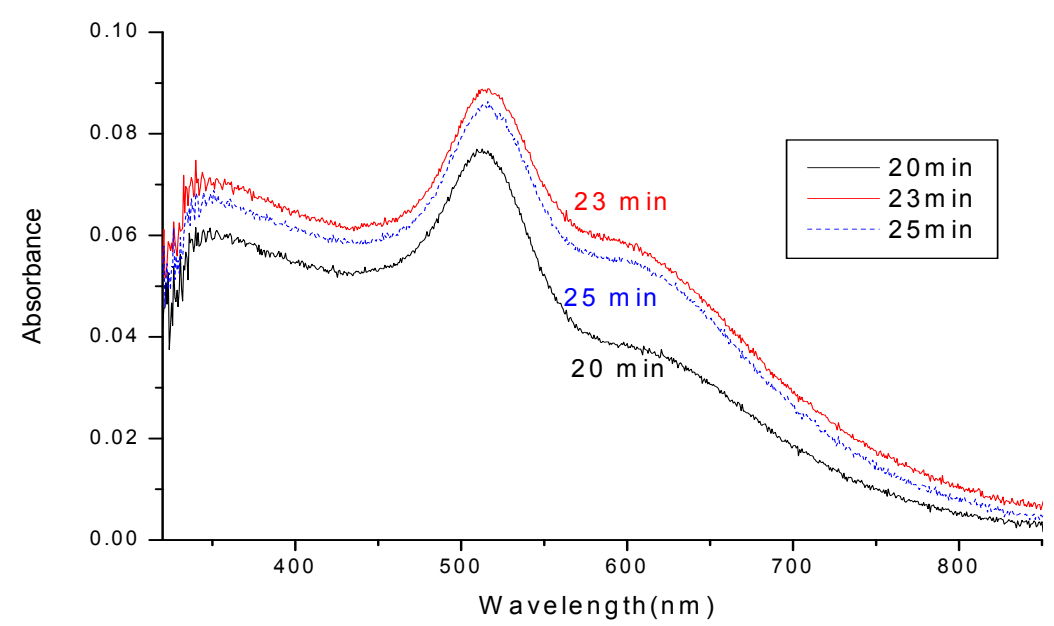

Figure 5. Absorption spectrum of $\mathrm{CM}_{\mathrm{Au}}$ 's on glass substrate with three different self-assembled time.

\section{Results and Discussion}

Thermal characteristics of the LPFG grating such as temperature and strain were first studied. The effects of temperature changes and strain variations must be avoided or compensated in order to accurately determine small variations in concentrations or RI. The resonance wavelength of the LPFG grating used here was shifted to the longer wavelength when temperature is increased. Using a temperature-controllable chamber the temperature sensitivity of the LPFG was determined by measuring the wavelength shift of the attenuation bands as a function of temperature, which was carried out by heating the gratings from 30 to $100{ }^{\circ} \mathrm{C}$ in incremental steps of $10{ }^{\circ} \mathrm{C}$. The results of measured sensitivity for an LPFG and a $\mathrm{CM}_{\mathrm{Au}} \mathrm{LPFG}$ are shown in Figs. 6 and 7, respectively. Based on a linear fit to the data curves, the temperature sensitivity for the investigated LPFG was found to almost remain unchanged before and after the modification of the grating surface with colloidal gold nanoparticles, which is approximately $0.056 \mathrm{~nm} /{ }^{\circ} \mathrm{C}$, about six times larger than that of the FBG $(\sim 0.01$ $\mathrm{nm} /{ }^{\circ} \mathrm{C}$ ) made by UV irradiation method.

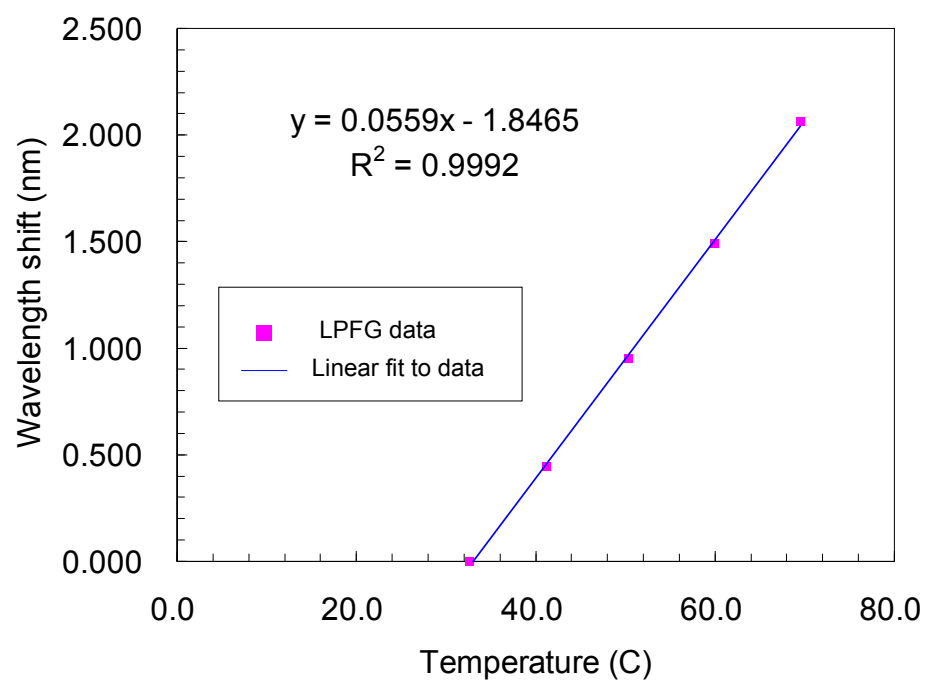

Figure 6. Wavelength shifts of a typical LPFG against temperature changes. 


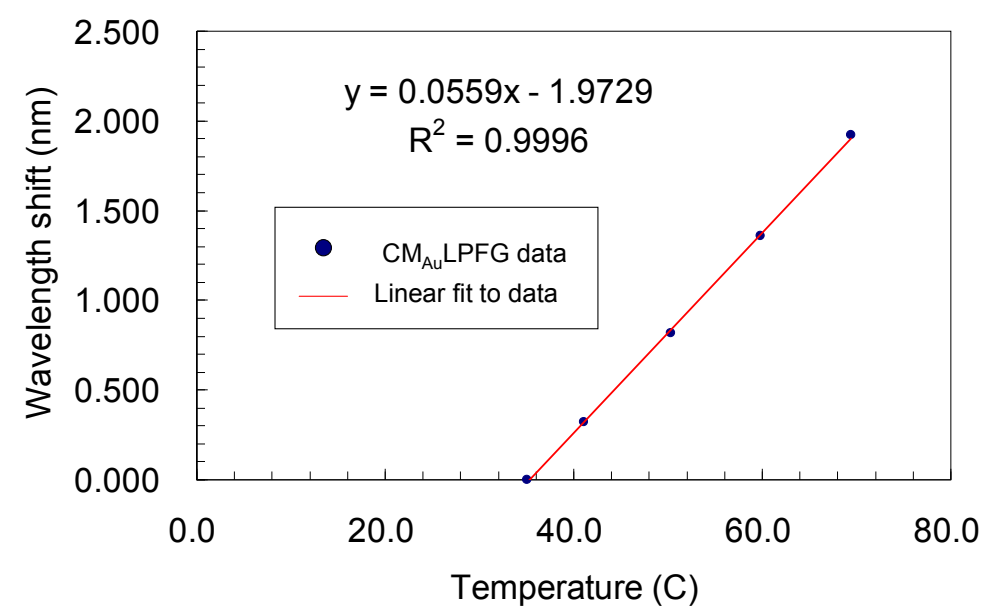

Figure 7. Wavelength shifts of the $\mathrm{CM}_{\mathrm{Au}} \mathrm{LPFG}$ versus temperature.

Strain-induced wavelength responses of LPFGs were found to be more dependent on the type of fiber. In particular, gratings written in Furukawa or Corning fibers exhibited nearly insensitive to strain variations. The strain sensitivity of the LPFG was determined by measuring the wavelength shift of the attenuation bands as a function of strain applied to the fiber, which was performed by mounting the grating on a translation stage that was moved outward to induce a strain in the optical fiber. The results for an LPFG and a $\mathrm{CM}_{\mathrm{Au}} \mathrm{LPFG}$ are shown in Figs. 8 and 9, respectively, which are approximately $\sim 0.13 \mathrm{pm} / \mu \varepsilon$ and $\sim 0.14 \mathrm{pm} / \mu \varepsilon$, respectively, indicating that strain sensitivity of the LPFG is unaltered by the influence of the presence of gold colloids applied on the grating surface. It can be seen that both temperature and strain sensitivities of the LPFG were not influence by the presence of a monolayer of gold colloids on grating region.

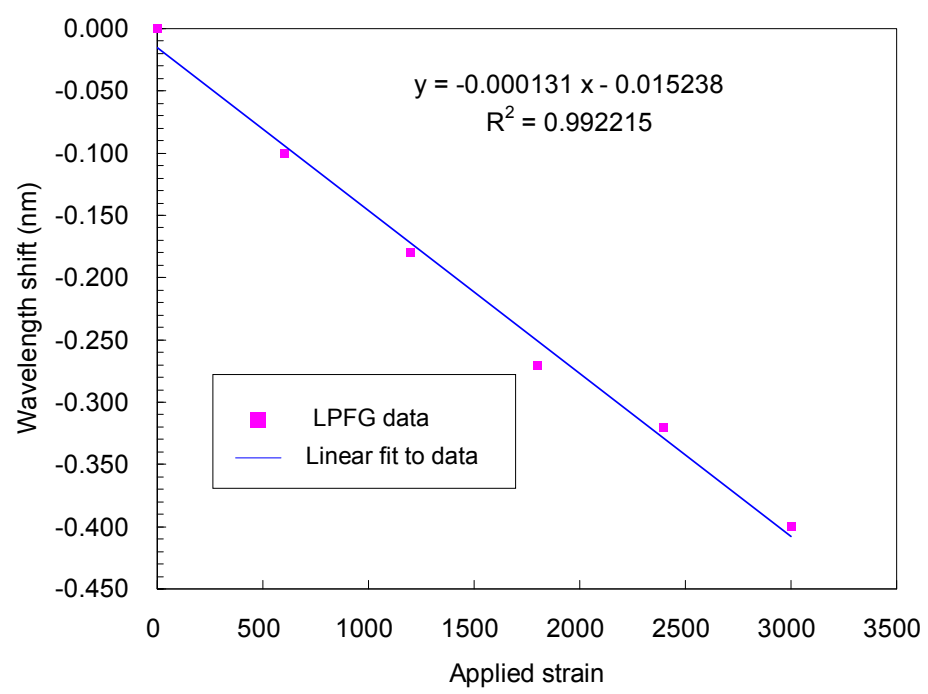

Figure 8. Wavelength shifts of the LPFG versus strain. 


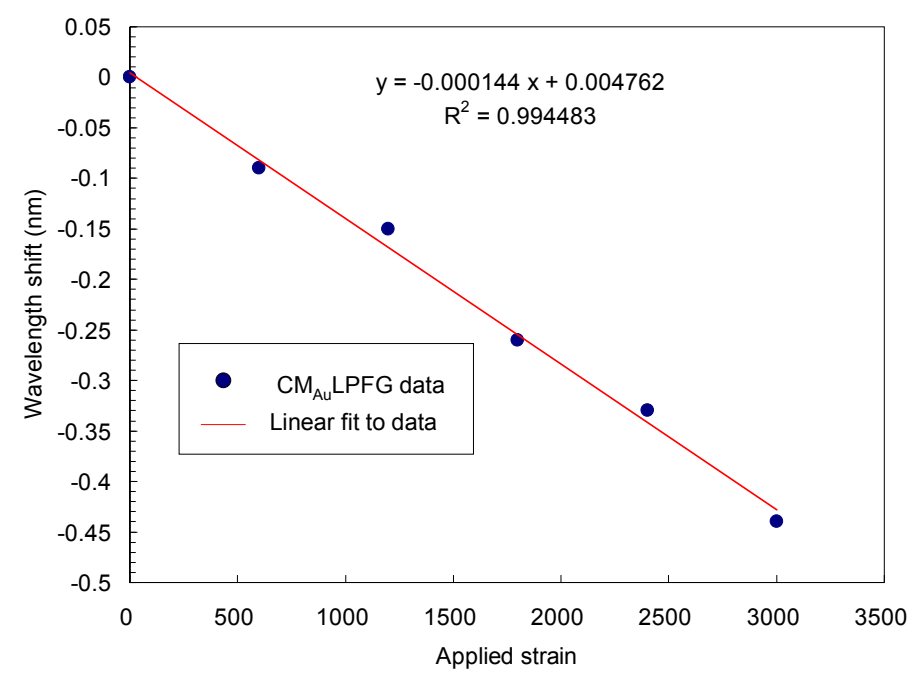

Figure 9. Wavelength shifts of the $\mathrm{CM}_{\mathrm{Au}} \mathrm{LPFG}$ versus strain.

In the experiment the strain sensitivity of all LPFG gratings used in this work were very small $(\sim 0.13 \mathrm{pm} / \mu \varepsilon)$ and they exhibited nearly insensitive to strain variations. For temperature variations of $1{ }^{\circ} \mathrm{C}$, the temperature-induced wavelength shift of $0.06 \mathrm{~nm}$ was larger than that spectral resolution of $0.02 \mathrm{~nm}$ used in our spectrum analyzer. For precise measurement, experimental setup and sample solution were kept at the same temperature (within $0.1^{\circ} \mathrm{C}$ ). During the course of measurement the fiber was maintained straight to avoid any deformation induced on the LPFG. Therefore, the results reported here were not influenced by both temperature and strain effects. To illustrate the application of an LPFG as a RI sensor of a concentration sensor, measurements with sucrose and sodium chloride aqueous solutions were performed. The surrounding RI was controlled through the use of sucrose solutions with various concentrations. As shown in Fig. 10, the relationship between RI and sucrose concentration has been determined and shown to be linear [19]. Experimental results for both solutions show that the LPFG-based sensors exhibited a linear decrease in the transmission loss and resonance wavelength shift when the concentration increased, demonstrating that the LPFG and $\mathrm{CM}_{\mathrm{Au}} \mathrm{LPFG}$ gratings are suitable for chemical sensing.

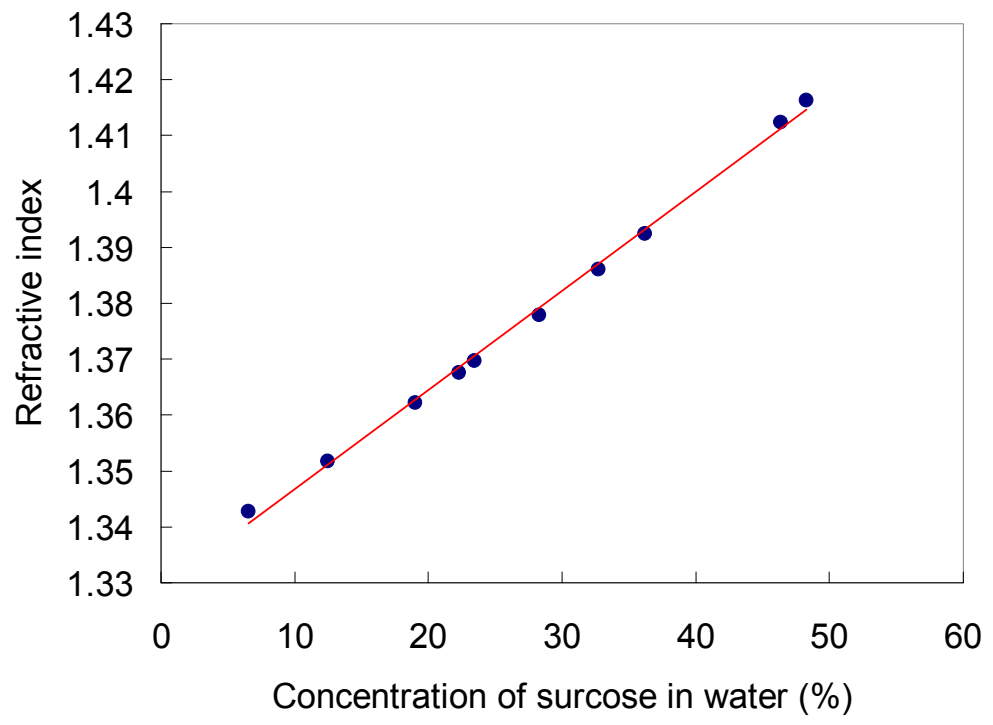

Figure 10. A plot of solution refractive index versus concentration of sucrose in the solution. 
To study the influence of the gold nanoparticles on the performance of the LPFG as a chemical concentration sensor, the spectral response of an LPFG fabricated in Furukawa SM-332 fiber to immersion in solution of varying concentration of sucrose was measured before and after the deposition of a layer of gold colloids. Fig. 11 shows wavelength shifts of a typical LPFG sensor against sucrose concentration and, hence, the corresponding changes in surrounding RI. When the concentration and, hence, the RI of a sucrose solution increased in the range of 1.34-1.39, the transmission spectrum of the LPFG sensor exhibited a decrease in the transmission loss at the resonance wavelength, and a blue shift in the resonance wavelength. Figs. 12 and 13 show a linear fit to the plot of peak wavelength as a function of refractive index $n$ for an LPFG and a $\mathrm{CM}_{\mathrm{Au}} \mathrm{LPFG}$, respectively. The resonance wavelength of $1584.72 \mathrm{~nm}$ for the LPFG in air was chosen because the maximum sensitivity was found at that wavelength. A linear regression approach was employed to calculate the RI sensitivity of each LPFG sensor. Our analysis determined that the RI sensitivity of the LPFG and $\mathrm{CM}_{\mathrm{Au}} \mathrm{LPFG}$ were $-59.9 \mathrm{~nm} /$ refractive index unit (RIU) and $-80.4 \mathrm{~nm} / \mathrm{RIU}$, respectively, which leads to a limit of detection ( $\mathrm{LOD}=3 \sigma / \mathrm{m}, \sigma=$ standard deviation of transmission loss peak in measuring the blank sample, $m=$ slope) in index, $1.0 \times 10^{-3}$ and $7.4 \times 10^{-4}$, respectively. For one unit change in the RI to external medium, the wavelength shift of the LPFG with gold colloids is increased by $\sim 20.5 \mathrm{~nm}$. It can be seen that a noticeable increase in RI spectral sensitivity was obtained when the grating portion of the LPFG was modified by self-assembled gold colloids.

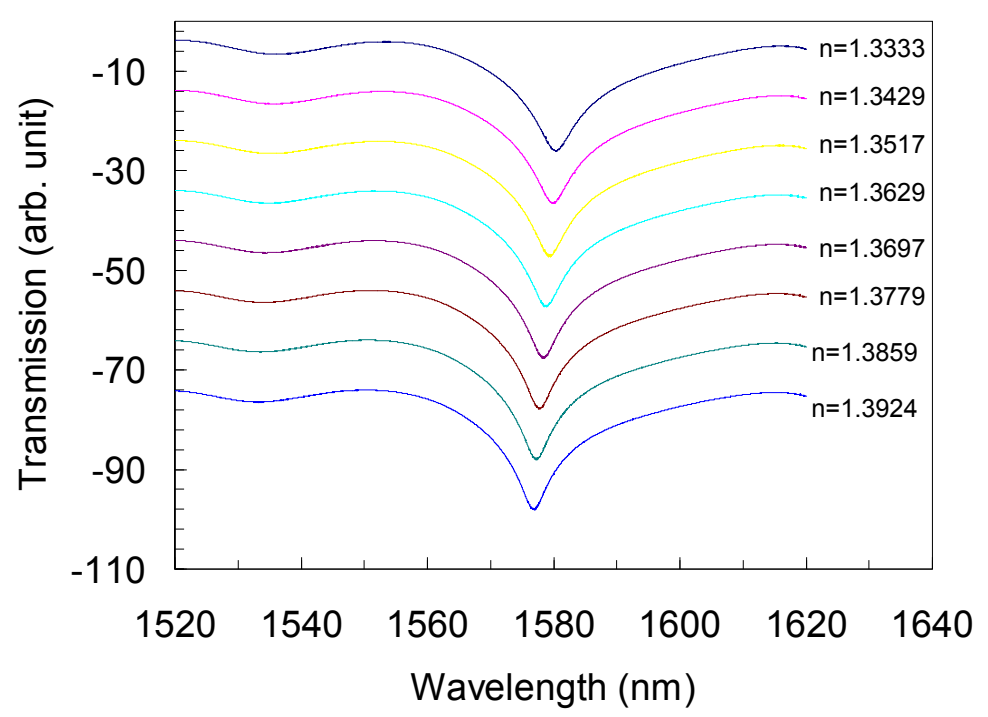

Figure 11. Transmission spectra of a typical LPFG in sucrose aqueous solution with increasing weight concentration. 


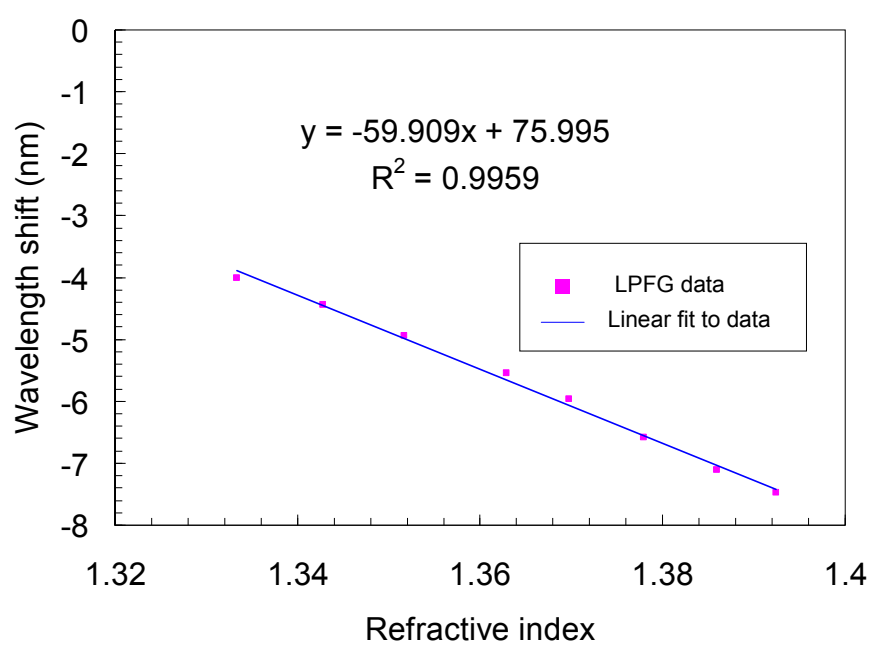

Figure 12. Wavelength shift of a bare LPFG sensor with increasing concentration of sucrose in aqueous solution.

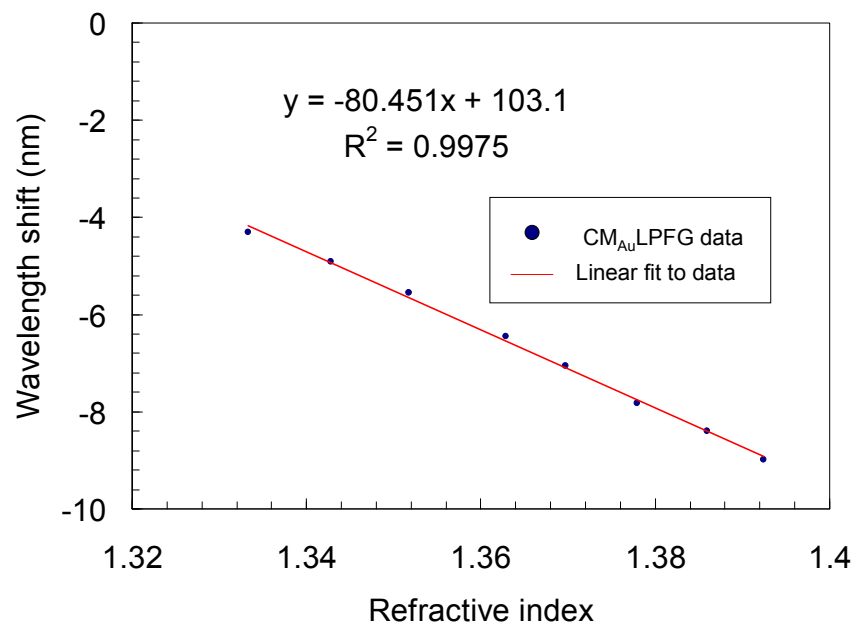

Figure 13. Wavelength shift of a $\mathrm{CM}_{\mathrm{Au}} \mathrm{LPFG}$ sensor with increasing concentration of sucrose in aqueous solution.

This $\mathrm{CM}_{\mathrm{Au}} \mathrm{LPFG}$ sensor is based on the principle that the surface plasmon resonance is highly sensitive the index of refractive at the interface between the cladding layer and a sample upon the gold surface. The sensitivity of the LPFG arises from the fact that the effective RI of a cladding mode can be modified by the RI of the surrounding medium. Thus the large enhancement of RI sensitivity for the LPFG surface-modified fiber is mainly due to the localized surface plasmons of gold nanospheres caused an increase in the local electromagnetic field (evanescent wave) strength for the surrounding analyte molecules. As mentioned in our previous work of Ref. 17, the sensitivity can be enhanced by the optimization of some key parameters, such as the size and density of immobilized gold colloids, surface structure of the functionalized monolayer, length of the LPFG fiber for immobilization of gold colloids and detection wavelength. In this study, we have optimized the size and density of immobilized gold colloids by controlling self-assembled time of colloidal gold monolayer and using a longer detection wavelength for LPFG. The self-assembled time and detection wavelength used in previous work were $20 \mathrm{~min}$ and $1524.5 \mathrm{~nm}$, respectively, compared with those of $23 \mathrm{~min}$ and 1584.72 $\mathrm{nm}$ in present work. It can be seen that the increase in RI spectral sensitivity for this surface-modified 
LPFG sensor is $20.5 \mathrm{~nm} / \mathrm{RI}$ (from $-59.9 \mathrm{~nm} / \mathrm{RI}$ to $-80.4 \mathrm{~nm} / \mathrm{RI}$ ) in present work and $5.52 \mathrm{~nm} / \mathrm{RI}$ (from $17.93 \mathrm{~nm} / \mathrm{RI}$ to $-23.45 \mathrm{~nm} / \mathrm{RI}$ ) in previous work [17], respectively, showing about a factor of four enhancement for RI spectral sensitivity.

The RI sensitivity of an LPFG to concentration change in chemical solution was further investigated using sodium chloride $(\mathrm{NaCl})$ solutions. The LPFG under test was fabricated in a Corning SM-28 fiber and the temperature kept constant to $0.1^{\circ} \mathrm{C}$. The results in Fig. 14 show that the LPFG also exhibited a linear decrease in resonance wavelength shift when the concentration of chloride ions increased. Based on the same analysis technique the measured spectral sensitivity of the LPFG sensor in terms of $\mathrm{NaCl}$ weight concentration increased from $-0.05 \pm 0.01(\mathrm{~nm} / \%)$ to $-0.07 \pm 0.01(\mathrm{~nm} / \%)$; while for LOD of this type of sensor, our results show a twofold enhancement in weight concentration, from $0.04 \pm 0.01 \%$ to $0.02 \pm 0.01 \%$. On the other hand, when conducting the same tests, experimental result for the accuracy of concentration measurement for chloride ions with this gold-coated LPFG sensor also shows a noticeable increase, which was better than $0.2 \pm 0.1 \%$ by weight concentration, about a three times increase, comparing to a bare LPFG sensor which has a measurement accuracy of $6.0 \pm 0.1 \%$. A weight concentration standard of $17.5 \%$ solution was used to determine the accuracy of LPFG sensor. Twenty measurements were taken and the concentration values were calculated using the linear fit function. The accuracy of concentration measurement is determined by using rms concentration value obtained from the prediction of linear fit and the standard. Therefore, results presented here demonstrate that the presence of a layer of colloidal gold nanospheres on the grating region could increase its sensitivity in detecting solution concentrations significantly.

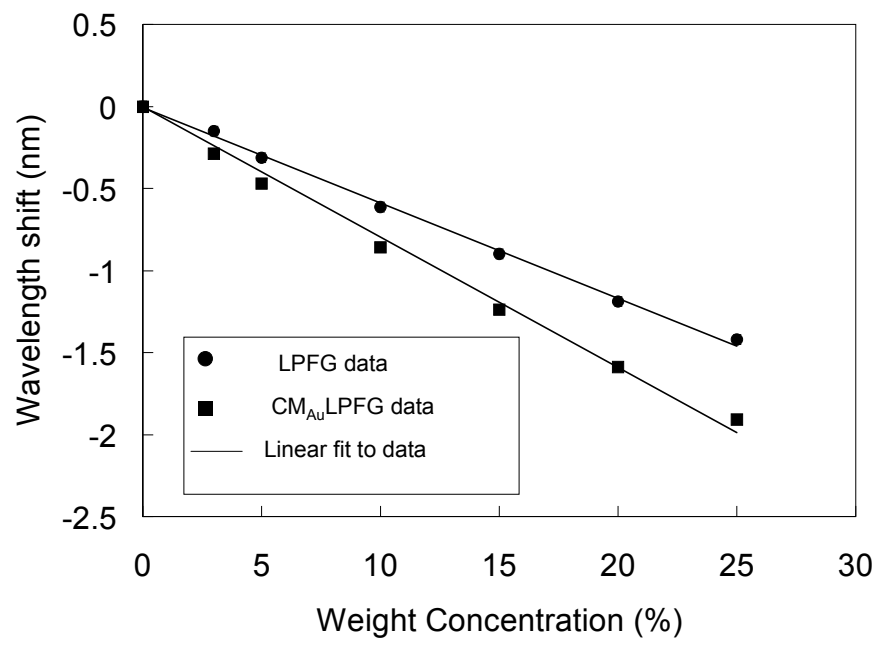

Figure 14. Wavelength shift of a bare LPFP sensor (black squares) and a $C_{\mathrm{Au}} L P F G$ sensor (black circles) in sodium chloride aqueous solution with increasing weight concentration.

\section{Conclusion}

In conclusion, a simple and sensitive optical fiber chemosensor relying on the modification of the grating portion of an LPFG with aqueous solution of chemicals that can significantly enhance the sensitivity and detection limit for chemical solutions is presented. The realization of the sensors is through the measurement of resonance wavelength shift in transmission spectra of the sensing fiber grating. We report on the sensing characteristics (temperature, strain, RI, and concentration) for this 
type of LPFG sensor with and without the modification of a thin layer of colloidal gold nanoparticles on the grating region. Enhanced sensitivity to surrounding RI with the presence of the colloidal gold layer was observed. Our results presented here demonstrate that the LPFG fiber sensor can provide a RI resolution of $\sim 10^{-3}$ to $10^{-4}$ for refractive indices in the range of 1.34 to 1.39 , suggesting that these devices may be suitable for use with aqueous solutions for chemical sensing. This type of sensor has potential applications in medical diagnostics, biochemical sensing, and environmental monitoring. The advantage of this type of the sensor is relatively simple of construction, compact, light, robust, lowcost, and ease of use. Moreover, the sensor has the potential capability for on-site, in vivo, and remote sensing, and has the potential use for smart structure and material of fiber communications and disposable sensors.

\section{Acknowledgements}

This work was partially supported by the National Science Council (NSC) of Taiwan under Contract Nos. NSC 95-2120-M-194-003, NSC-94-2120-M-194-006, NSC 94-2112-M-194-016, NSC 93-2111E-224-022, NSC 94-2111-E-224-026, and NSC 95-2221-E-224-066.

\section{References and Notes}

1. Vengsarkar, A. M.; Lemaire, P. J., Judkins, J. B.; Bhatina, V.; Erdogan, T.; Sipe, J. E. Longperiod fiber gratings as band-rejection filters. IEEE J. Lightwave Technol. 1996, 14, 58-65.

2. Vengsakar, A.M; Pedrazzani, J.R.; Judikins, J.B.; Lemaire, N.S.; Bergano, N.S.; Davidson C.R. Long-period fiber-grating based gain equalizers. Opt. Lett. 1996, 21, 336-338.

2. Das, M.; Thyagarajan, K. Dispersion compensation in transmission using uniform long period fiber gratings. Opt. Commun. 2001, 197, 159-163.

3. Abramov, A.A.; Hale, A.; Windeler, R.S.; Strasser, T.A. Widely tunable long-period fibre gratings. Electron Lett. 1999, 35, 81-82.

4. Chiang, K.S.; Liu, Y.; Ng, M.N.; Li, S. Coupling between two parallel long-period fibre gratings. Electron. Lett. 2000, 36, 1408-1409.

5. Bhatia, V.; Campbell, D.; Claus, R.O.; Vengsarkar, A.M. Simultaneous strain and temperature measurement with long-period gratings. Opt. Lett. 1997, 22, 648-650.

6. Bhatia, V.; Vengsarkar, A. M. Optical fiber long-period grating sensors. Opt. Lett. 1996, 21, 692-694.

7. Bhatia, V.; Campbell D.K.; Sherr D.; Tiffanie, G.D.; Noel, A.Z.; Gregory, A.; Kent, A.M.; Clau, R.O. Temperature-insensitive and strain-insensitive long-period gratings sensor for smart structures. Opt. Eng. 1997, 36, 1872-1876.

8. Bhatia, V.; Tiffanie, G.D.; Noel, A.Z.; Clau, R.O. Temperature-insensitive long-period grating for strain and refractive index sensing. Proc. SPIE. 1997, 3042, 194-202.

9. Bhatia, V. Applications of long-period gratings to single and multi-parameter sensing. Opt. Express. 1999, 4, 457-466.

10. Allsop, T.; Zhang L.; Bennion I. Detection of organic aromatic compounds in paraffin by a long period fiber grating optical sensor with optimized sensitivity. Opt. Commun. 2001, 191, 181-190. 
11. James S.; Tatam, R. Optical fibre long-period grating sensors: characteristics and application. Meas. Sci. Technol. 2003, 14, R49-R61.

12. Patrick, H. J. ; Kersey A. D.; Bucholtz, F. Analysis of the response of long period fiber gratings to external index of refraction. J. Lightwave Technol. 1998, 16, 1606-1612.

13. Okamoto, T.; Yamaguchi I.; Kobayashi, T. Local plasmon sensor with gold colloid monolayers deposited upon glass substrates. Opt. Lett. 2000, 25, 372-374.

14. Nath, N.; Chilkoti, A. A colorimetric colloidalbgold sensor to interrogate biomolecular interactions in real-time on a surface. Anal. Chem. 2003, 4, 504-509.

15. Cheng, S.-F.; Chau, L.-K. Colloidal Gold-Modified Optical Fiber For Chemical and Biochemical Sensing. Anal. Chem. 2003, 75, 16-21.

16. Takei, H.; Himmelhaus, M.; Okamoto, T. Absorption spectrum of surface-bound cap-shaped gold particles. Opt. Lett. 2003, 27, 342-344.

17. Tang, J.L.; Cheng, S.F.; Hsu, W.T.; Chiang, T.Y.; Chau, L.K. Fiber Optic Biochemical Sensing with a Colloidal Gold-Modified Long Period Fiber Grating. Sensors \& Actuators: B. Chemical 2006, 119, 105-109.

18. Davis, D.D. ; Gaylod, T.K. ; Glytsis, E.N. ; Kosinski, S.G. ; Mettler S.C. ; Vengsarkar, A.M. Long-period fiber grating fabrication with focused $\mathrm{CO}_{2}$ laser pulses. Electron. Lett. 1998, 34, 302-303.

19. Hwang, T.-C. Experiments in Physical Chemistry, Gaulih Book Co., Taipei, 1994.

(C) 2008 by MDPI (http://www.mdpi.org). Reproduction is permitted for noncommercial purposes. 